CHERCHER, REPÉRER, AVANCER

\title{
« LA TRADUCTION EST AU CEUR DE MON TRAVAIL DE COMPARATISTE »
}

Entretien avec James Q. Whitman, réalisé par Guillaume Richard et Lionel Zevounou

Lextenso | « Droit et société »

2019/1 N 101 | pages 101 à 113

ISSN 0769-3362

ISBN 9782275029337

Article disponible en ligne à l'adresse :

https://www.cairn.info/revue-droit-et-societe-2019-1-page-101.htm

Distribution électronique Cairn.info pour Lextenso.

(C) Lextenso. Tous droits réservés pour tous pays.

La reproduction ou représentation de cet article, notamment par photocopie, n'est autorisée que dans les limites des conditions générales d'utilisation du site ou, le cas échéant, des conditions générales de la licence souscrite par votre établissement. Toute autre reproduction ou représentation, en tout ou partie, sous quelque forme et de quelque manière que ce soit, est interdite sauf accord préalable et écrit de l'éditeur, en dehors des cas prévus par la législation en vigueur en France. Il est précisé que son stockage dans une base de données est également interdit. 


\section{Question en débat}

Entretien avec James Q. Whitman 


\title{
« La traduction est au cœur de mon travail de comparatiste »
}

\author{
Entretien avec James Q. Whitman * \\ réalisé par Guillaume Richard et Lionel Zevounou
}

À l'occasion de la parution de son dernier ouvrage ${ }^{1}$, il a paru utile de revenir sur les travaux de James Q. Whitman, professeur d'histoire du droit et de droit comparé à l'Université de Yale. Le professeur Whitman fait partie des quelques universitaires américains à s'intéresser aux systèmes juridiques continentaux que sont la France, l'Allemagne ou l'Italie. Restituer le regard qu'il porte sur sa double pratique d'historien et de comparatiste apparaît pertinent dans le contexte français de cloisonnement relativement avancé des savoirs disciplinaires, alors même que l'avenir disciplinaire de l'histoire du droit semble discuté. Le travail de James Whitman enrichit la pratique du droit comparé, bien souvent réduite à une simple évaluation de différents systèmes juridiques sous forme de benchmarking. Donner la parole à James Whitman incite à une ouverture du droit vers d'autres disciplines.

Une telle ouverture disciplinaire se justifie d'autant mieux que l'on observe dans le champ des sciences sociales un retour de travaux pluridisciplinaires portant sur la race. Comme le montre avec conviction son dernier ouvrage, les juristes de la première moitié $d u X X^{e}$ siècle ont, à leur niveau, contribué à la naturalisation du paradigme racial : le droit racial américain a servi de référence positive aux juristes nazis dans l'élaboration des lois de Nuremberg en 1935. La focalisation sur la race n'a pas été seulement le fruit des grandes puissances coloniales et le système juridique américain a aussi apporté sa pierre à cet édifice, tant au regard des lois sur la ségrégation raciale que des prétentions impériales de la puissance américaine émergente. Au fond, ce à quoi invite Le modèle américain d'Hitler ${ }^{2}$, c'est bien à porter un regard juridique global sur la manière dont a été pensée la race tout au long des XIX et XX siècles.

Pouvez-vous retracer votre parcours et la façon dont vous vous êtes intéressé à l'histoire du droit?

Mon parcours, marqué par l'obtention d'un doctorat (Ph.D. en histoire intellectuelle), est de plus en plus fréquent dans les facultés de droit américaines. Pendant

\footnotetext{
* Cet entretien a également été publié dans le n 4, 2018 des Cahiers Jean Moulin, <https://revues.univlyon3.fr/cjm/index.php?id=575>.

1. James Q. Whitman, Hitler's American Model, Princeton : Princeton University Press, 2017, (trad. française : Le modèle américain d'Hitler. Comment les lois raciales américaines inspirèrent les Nazis, Paris : Armand Colin, 2018).

2. Ibid.
} 
longtemps, l'enseignement du droit anglo-américain a été le monopole d'une bourgeoisie de praticiens. Le diplôme exigé pour l'enseignement était le J.D., délivré par les law schools après l'obtention d'un premier diplôme généraliste ; il était complété par un stage pratique. J'ai accompli moi-même un stage comme clerk (assistant de justice) pendant une année. Mais à partir des années 1970, la possession du diplôme de doctorat en plus du J.D. est devenu une exigence de plus en plus fréquente dans la discipline juridique, sous l'influence (exercée sur toutes les sciences sociales) de l'économie.

Mon intérêt pour l'histoire du droit vient d'abord d'une admiration ancienne pour la tradition intellectuelle allemande. Mes premières recherches ont porté sur l'Antiquité et le droit romain, à partir de l'approche allemande de l'Altertumswissenschaft. Sous la direction d'Arnaldo Momigliano, ma thèse de doctorat a porté sur l'influence jouée par le droit romain sur la culture juridique allemande au XVIII et au XIX ${ }^{\mathrm{e}}$ siècle $^{3}$. L'idée était alors de montrer le rôle joué par le droit romain au moment du développement industriel du XIX ${ }^{\mathrm{e}}$ siècle. Le droit romain attirait les juristes allemands comme moyen de placer le droit au-dessus de toute forme de conflit politique dans un contexte de fondation d'une cohésion nationale. L'une des raisons pour lesquelles mes travaux ont été admis dans le champ académique américain vient aussi de l'importance reconnue au rôle du droit allemand. La culture latiniste a aussi occupé une place importante aux États-Unis, certes moins centrale qu'en Allemagne; le droit romain était également enseigné dans les facultés de droit américaines au XIX siècle.

La discipline juridique comprend en France trois sections : droit privé, droit public et histoire du droit. Ce tripartisme engendre une division du travail disciplinaire assez tranchée. Qu'en est-il aux États-Unis et quel est l'état de la recherche en histoire du droit au sein de la discipline juridique?

La distinction entre droit privé et droit public n'existe pas en tant que telle aux États-Unis. La spécialisation ne prend pas les mêmes chemins qu'en Europe, puisqu'il n'existe pas à proprement parler de chaire exclusivement dédiée à l'histoire du droit. Le spécialiste d'histoire du droit n'est pas cantonné à cette matière et son enseignement ne porte jamais exclusivement sur l'histoire du droit. Bien plus, la plupart des historiens du droit aux États-Unis ne sont pas nommés en raison de cette qualité, mais parce qu'ils sont aussi capables d'enseigner des matières qui ne relèvent pas de l'histoire du droit. J'ai commencé à enseigner le droit des faillites pour ma part.

En général, le regard porté sur l'histoire du droit aux États-Unis est celui d'une discipline très intellectuelle, respectée comme telle par les pairs. Au sein de l'histoire du droit, orientée principalement vers l'histoire du droit américain, l'histoire du droit constitutionnel comparée est celle qui attire le plus d'étudiants américains. L'histoire du droit administratif, du droit des contrats et du droit pénal ne sont pas non plus en reste dans les matières enseignées.

3. ID., The Legacy of Roman Law in the German Romantic Era: Historical Vision and Legal Change, Princeton: Princeton University Press, 1990. 
La traduction occupe un rôle majeur dans votre travail. Le soin pour rendre accessibles au lectorat anglophone des textes allemands ou latins, selon vos ouvrages, en constitue la meilleure preuve. Quelle importance revêt ce souci de traduction dans le contexte où vous évoluez, celui du droit anglo-saxon dont le modèle et le monolinguisme affirmé sont aujourd'hui dominants à l'échelle mondiale?

La traduction est au cœur de mon travail de comparatiste, afin de rendre accessibles les traditions juridiques européennes au public universitaire américain et, inversement, de faciliter l'accès de textes juridiques américains à l'Europe. Si l'on élargit la perspective, on peut dire que la traduction - au sens littéral et au sens philosophique - est au cœur de mon travail de comparatiste. Le travail de la science constitue aussi, dans une certaine mesure, un travail de traduction. C'est en traduisant que l'on finit par gagner en autonomie de pensée. L'un des ressorts de l'attractivité juridique de la culture allemande au XIX ${ }^{e}$ siècle tient au remarquable travail de traduction de la culture latine pour l'expliquer à d'autres cultures juridiques. La tradition juridique japonaise est aussi marquée par ce même trait distinctif.

La culture juridique américaine est une culture très monolingue. Dans les années 1960 et 1970, les États-Unis étaient encore relativement ouverts aux autres langues. La liste des cours durant les années 1960 dans les facultés de droit laissait une place significative à des professeurs étrangers et à l'ouverture vers les traditions juridiques européennes. Mais ce mouvement s'est aujourd'hui refermé, pour trois raisons. La première tient à l'essor et à l'influence de la science économique; le mouvement Law and Economics a contribué à couper les liens de la culture juridique américaine avec d'autres traditions doctrinales encore en vigueur en France par exemple. Ces traditions sont regardées avec une certaine condescendance du côté américain, où beaucoup croient détenir avec le mouvement Law and Economics la nouvelle "vérité » sur le droit. La seconde raison tient à l'émergence des États-Unis comme seule superpuissance mondiale sur le plan géopolitique après la fin de la Guerre froide. La chaire universitaire que j'occupe, financée par la fondation Ford, est née dans les années 1950 de la volonté de comparer le système juridique américain avec les systèmes juridiques socialistes et européens continentaux. Il fallait comprendre les différences et si possible, s'agissant de l'Europe, chercher à les aplanir vis-à-vis du système juridique américain. De plus en plus désormais, les études comparatives sont intégrées aux "Area Studies», lesquelles relèvent davantage de la science politique que des facultés de droit. La troisième raison tient à l'émergence du droit constitutionnel américain. Les juristes américains croient, en dépit de leurs problèmes de politique intérieure récurrents, en la supériorité du modèle constitutionnel américain et ne s'intéressent guère aux modèles étrangers.

Vos travaux précédents ont porté sur l'origine du procès criminel de common law ${ }^{4}$, mais aussi sur le rôle des batailles rangées dans la résolution des conflits ${ }^{5}$. Votre propos consiste souvent à partir d'une question juridique nettement délimitée

4. ID., The Origins of Reasonable Doubt: Theological Roots of the Criminal Trial, New Haven :Yale University Press, 2008.

5. ID., Verdict of Battle: The Law of Victory and the Making of Modern War, Cambridge : Harvard University Press, 2012. 
pour prendre à revers l'opinion la plus couramment partagée et à tisser des liens apparemment paradoxaux : par exemple entre les règles du procès criminel anglosaxon et ses origines théologiques, en remontant de la période contemporaine à la période médiévale, ou, de façon plus synchronique, en montrant dans votre dernier ouvrage des connexions entre le droit de l'Allemagne nazie et celui de l'Amérique du début du XX' siècle6. Quel objectif cette manière d'aborder les choses poursuitelle? S'agit-il de proposer une forme d'écriture qui puisse assurer une diffusion plus large des recherches en histoire du droit, y compris dans le débat public? Ou cela traduit-il aussi plus profondément la volonté d'une reconstruction généalogique des faits historiques, afin de montrer qu'ils ne sont pas linéaires?

La réponse est complexe. L'un des dangers qui guettent la science, la science juridique en particulier, est de ne s'adresser qu'à un public de spécialistes. C'est ce que j'ai tenté d'éviter dans mon dernier livre. L'un des avantages d'être professeur dans une faculté comme Yale est de pouvoir présenter toutes les semaines ses recherches aux autres collègues. Cette incitation pousse à parler de sujets aussi ardus que le droit au Moyen Âge ou le droit allemand à un public de non-spécialistes. Je trouve que cette incitation est stimulante.

La réputation de mon maître Momigliano tenait à cette manière de faire les choses. Juif italien chassé en 1938 à cause des lois raciales, il s'est exilé aux ÉtatsUnis et a trouvé un poste à l'université de Chicago. Cet exil l'a poussé à présenter sa pensée, en particulier les concepts et schèmes de pensée du droit italien aux collègues anglais ; cela a stimulé des découvertes intellectuelles de grande envergure. Certes, je ne suis pas Momigliano, mais présenter ses recherches aux collègues spécialistes de Law and Economics, de droit de la concurrence ou de droit des contrats nécessite au préalable un grand travail de traduction - on y revient - qui oblige à formuler sa pensée d'une manière à intéresser des spécialistes d'autres matières.

Mais cet effort ne garantit pas de trouver un large public: mon livre Verdict of Battle $^{7}$ n'a par exemple recueilli qu'un écho limité, sans doute en raison de son contenu malgré tout trop savant.

Quant à la conception de l'histoire que mes écrits impliqueraient, je ne crois pas me retrouver dans une quelconque approche philosophique à l'instar d'un Foucault et de ses procédés généalogiques. Ce que je tente de montrer, c'est le sens des traditions profondes qui innervent la pensée juridique. Par exemple, l'analyse que je fais du principe procédural du "doute raisonnable " (reasonable doubt) 8 montre qu'à l'origine ce principe s'ancre dans la théologie et non dans le droit. Il n'avait rien à voir à l'origine avec la question de la preuve.

Mais c'est en reconnaissant à quel point on est victime de son histoire que l'on prend la mesure de l'importance et du sens profond des normes juridiques. Les juristes ambitionnent généralement de résoudre les problèmes sociaux. La pensée juridique allemande du XIX ${ }^{\mathrm{e}}$ siècle est à cet égard caractéristique d'une telle approche. Le récit de

\footnotetext{
6. ID., Hitler's American Model, op. cit.

7. ID., Verdict of Battle: The Law of Victory and the Making of Modern War, op. cit.

8. ID., The Origins of Reasonable Doubt: Theological Roots of the Criminal Trial, op cit.
} 
la pensée juridique est au fond un récit où les juristes pensent pouvoir apporter des solutions aux problèmes sociaux par le moyen de la technique juridique; mais, à chaque fois, les solutions qu'ils proposent s'avèrent pour le moins insatisfaisantes. Mon propos cherche à montrer ce qui a animé au départ la construction des systèmes techniques censés résoudre les problèmes sociaux auxquels on a voulu s'attaquer, et finalement ce qui a entraîné le plus souvent l'échec de ces solutions.

Même si je partage avec Foucault le refus d'une «raison" de l'histoire, j'ai des désaccords avec sa pensée, notamment son histoire de la peine qui m'apparaît erronée sur plusieurs points. Bien sûr, cela n'enlève rien au génie du personnage ; mon problème porte davantage sur les disciples de Foucault et l'usage parfois discutable de sa pensée, en particulier l'approche consistant à inscrire la pensée juridique dans des métarécits globalisants en perdant de vue certaines spécificités historiques. Mon approche est différente : je tente d'identifier un problème pour voir où il me mène. Je ne cherche pas à l'inscrire dans une histoire globale. Par exemple, le droit civilisé de la guerre date du XIX ${ }^{e}$ siècle. Or, depuis cette époque, les guerres sont beaucoup plus meurtrières. Comment expliquer ce paradoxe ? C'est ce que j'appelle un problème.

L'identification de ces problèmes part généralement des États-Unis, parce que mon travail est un travail situé. L'écriture de mon livre sur le droit de la guerre ${ }^{9}$ a été stimulée par le second conflit irakien. Le discours dominant était d'affirmer qu'il s'agissait d'une victoire. Mais pouvait-on parler véritablement de victoire ? À voir les désastres humanitaires engendrés par ce conflit, on peut sérieusement en douter. Il existait, au XVIII siècle, un droit de la victoire : un ensemble de normes qui codifiaient, au terme d'un conflit, le camp des gagnants et celui des perdants. De nos jours, identifier chacun de ces camps n'est pas une question facile à résoudre, d'autant moins si l'on prend l'exemple du second conflit irakien. Il est impossible de déterminer clairement ce qu'a gagné le "vainqueur». Ce type de guerre à vocation humanitaire se donne pour cause de rétablir une certaine idée de la «justice». Mais est-il jamais possible de rétablir cette justice dans tous ses aspects, surtout en matière de droits humains? C'est ce qui explique en partie que les tueries en Afghanistan et en Irak ne peuvent se terminer. Je ne crois pas que la mise en œuvre de l'idéal humanitaire au nom de la « justice », tel qu'on l'avait envisagé après le second conflit mondial, ait jamais pu triompher. Et l'on en revient finalement à ce travail inlassable des juristes qui développent et mettent en forme des techniques juridiques au service d'idéaux plus grands sans pouvoir en maîtriser par la suite les effets et les conséquences éventuelles.

Venons-en maintenant à votre dernier ouvrage sur le "modèle américain de Hitler " 10 . Les sciences sociales en France font de plus en plus appel à un travail dit de réflexivité, sur le modèle de ce qui se pratique outre-Atlantique. Quel rapport entretenez-vous avec votre sujet?

Comme beaucoup, j'ai pour le régime nazi une fascination horrifiée. Juifs polonais, mes grands-parents se sont réfugiés aux États-Unis au dernier moment, c'est-à-dire avant que les lois sur l'immigration renforcent le contrôle sur les immigrés juifs

9. ID., Verdict of Battle: The Law of Victory and the Making of Modern War, op. cit.

10. ID., Hitler's American Model, op. cit. 
d'Europe de l'Est. Nombre de demandeurs d'asile ont d'ailleurs été refoulés une fois la guerre intervenue. J'ai perdu onze personnes de ma famille dans les camps. Tout ceci a nourri mon intérêt pour la culture de l'Allemagne, d'abord pour sa musique, puis pour son droit et son histoire. La même culture qui a donné naissance à Brahms a aussi donné naissance à Hitler. Tout ceci explique pourquoi je dédie le livre aux "mânes de Louis B. Brodsky», auquel je m'identifie, comme mes parents avant moi ${ }^{11}$.

L'ouvrage mène une réflexion sur les phénomènes d'influence et d'échange d'institutions juridiques, dans une perspective de droit comparé. Il laisse pourtant peu de place apparente aux questions méthodologiques. Pour quelle raison? Et sachant qu'il existe plusieurs manières de parler et de faire du droit comparé, de laquelle vous réclameriez-vous?

J'ai pensé que des considérations méthodologiques diminueraient l'impact du livre. Dire que ce livre ne comprend que des faits ne signifie d'ailleurs rien en soi, puisque les faits sont toujours situés ou intégrés dans une pensée plus large. Du point de vue de l'efficacité rhétorique, l'idée était plutôt de faire état d'un problème juridique et de laisser le lecteur libre d'en faire ce qu'il voulait par la suite. Mon propos n'est pas non plus un ouvrage de vulgarisation à proprement parler. J'écris à un public dont je suppose qu'il est suffisamment averti. Les universitaires ont le temps de réfléchir. J'ai voulu proposer à un public qui en dispose moins un travail qui n'est pas de la vulgarisation, mais qui n'est pas non plus un travail purement académique.

La démarche de droit comparé suppose d'admettre que les solutions ou les raisonnements juridiques qui nous sont les plus communs ne sont pas les seuls possibles. L'enseignement américain est caractérisé par une tradition socratique: la forme des questions posées vise à interroger ce qui peut justifier telle ou telle règle. Le sous-entendu de la question est que la norme est généralement justifiée. Cette justification puise aujourd'hui principalement dans le mouvement Law and Economics. Elle suppose d'admettre qu'il n'y a généralement qu'une ou deux solutions possibles pour justifier une norme. C'est à partir de là qu'il faut ouvrir des possibilités en regardant vers l'Europe. En somme, le recours au droit comparé permet un décentrement du regard, généralement inattendu.

Prenons le cas du droit de la concurrence par exemple. On affirme généralement qu'il existe une différence entre la tradition américaine et européenne. Le droit américain de la concurrence est censé protéger la concurrence tandis que le droit européen protégerait les concurrents. La question est de savoir comment expliquer ces différences ? Elles tiennent principalement selon moi à des différences de culture et aux références axiologiques fondamentales avec lesquelles les deux droits travaillent. Le droit américain accorde une place importante au consommateur et à la demande ; le droit européen quant à lui met davantage l'accent sur le côté de l'offre. Ces deux modes de raisonnement se fondent sur des éléments culturels distincts 12 .

11. Mentionné au début de l'ouvrage (chapitre 1), le juge Brodsky a rendu une décision audacieuse jugeant qu'une manifestation s'opposant à la visite d'une délégation nazie en 1935 venue par bateau sur le SS Bremen et ayant conduit à souiller le drapeau nazi n'était pas contraire à la liberté d'expression.

12. James Q. Whitman, « Consumerism Versus Producerism: A Study in Comparative Law », The Yale Law Journal, 117 (3), 2007-2008, p. 340-406. 
Cette différence se donne à voir très clairement dans certains aspects du droit de la concurrence. On dit par exemple s'agissant de Uber que son entrée sur le marché doit faire baisser les prix en faveur des consommateurs là où, en Europe, les débats portent sur les effets délétères de l'introduction de ces grandes compagnies pour le secteur traditionnel des taxis. C'est seulement en étudiant le droit comparé que l'on comprend que ces différences axiologiques elles-mêmes sont fondées sur des intuitions peu démontrées ou sur des choix axiologiques s'imposant du fait du contexte culturel. La science économique n'est pas en mesure de dire au fond, qui, du consommateur ou du producteur a le plus perdu ou gagné. L'accent mis sur l'un ou l'autre de ces éléments tient à ces différences culturelles.

Votre ouvrage interroge les voies complexes de l'influence entre des droits ou des traditions juridiques différentes. Il montre comment certains traits du droit américain (la ségrégation, la discrimination raciale, l'interdiction des mariages interraciaux dans de nombreux États américains jusqu'aux années 1960) ont pu servir de référence, voire influencer les juristes nazis. Peut-on dire qu'il y a eu une influence du droit américain sur le droit nazi, en considérant l'influence comme l'emprunt fait à un droit étranger?

L'influence recouvre plusieurs choses: le désir de s'identifier, l'exploitation d'un emprunt fait à un modèle juridique étranger, l'attrait pour une culture juridique considérée comme prestigieuse. L'attrait des nazis pour les États-Unis tient aussi à des éléments de prestige, de rayonnement et de réputation. L'importation du modèle du droit contractuel américain en Europe se fonde aujourd'hui principalement sur des raisons liées au prestige économique. De la même manière hier, les nazis considéraient le rayonnement économique du système juridique américain et croyaient qu'il était en partie lié aux dispositifs perpétuant la ségrégation raciale : ils voyaient dans l'élément le plus conforme à leurs propres vues les raisons de l'influence américaine, y trouvant le moyen de confirmer la validité de leurs propres thèses. Il ne s'agissait pas d'interroger l'efficacité du système juridique et sa capacité à résoudre les antinomies normatives. L'influence ne s'explique pas par des relations de causalité. Elle réside dans les éléments supposés de puissance et l'attrait économique exercé par un ou plusieurs modèles étrangers. Au fond, et pour contourner cette représentation, je suis parti d'une étude beaucoup plus précise établissant la manière dont les nazis se sont représenté le droit américain.

\section{Les Critical Race Theories (CRT) devraient-elles s'inspirer de votre travail ?}

Il est assez difficile pour un homme blanc de répondre à cette question. J'imagine que ce que j'ai dit pourra servir de munition pour les travaux critiques futurs aux États-Unis. J'ai écrit quelques travaux à ce titre sur le droit comparé de la discrimination ${ }^{13}$. Le système juridique américain est souvent enclin à protéger la carrière, etc. En matière de discrimination, l'Europe se concentre plutôt sur la dignité. Cette différence est frappante. Les juristes européens sont souvent étonnés dès que

13. Gabrielle S. Friedman et James Q. Whitman, "The European Transformation of Harassment Law: Discrimination Versus Dignity », Columbia Journal of European Law, 9 (2), 2003, p. 241-274. 
l'on tente d'expliquer que ce qui est protégé dans le harcèlement sexuel aux ÉtatsUnis est moins la dignité en soi que les chances de carrières. Inversement, la dignité est une valeur très peu protégée aux États-Unis. Il est possible qu'un travail de droit comparé sur la race puisse conduire à mettre en lumière ce genre de différences.

La technique juridique joue un rôle particulier dans l'attrait des nazis pour le modèle américain, en particulier l'approche définitionnelle très large de la catégorie de race en droit américain. Cet emprunt permet aux nazis de se fonder sur l'argument d'autorité de l'emprunt afin d'économiser une définition complexe de la race.

Il faut insister sur le mot adapter plutôt qu'emprunter. Adapter au sens où la culture juridique allemande est tellement fière de ses traditions qu'elle n'aurait jamais décidé d'importer telle quelle la catégorie de race figurant dans le droit américain. Cela nous conduit à la différence entre les cultures dites " exportatrices » et les cultures "importatrices" en matière de droit comparé. Jusqu'à la fin du XVIII ${ }^{e}$ siècle, l'Italie a presque systématiquement été une culture importatrice. La culture juridique française au contraire demeure rétive aux traditions étrangères. Il en est de même des cultures juridiques allemande ou américaine, et ce, à la différence de la culture juridique canadienne qui fait la part belle aux autres cultures juridiques. Pour en revenir aux juristes nazis, ils n'auraient jamais copié tels quels les raisonnements juridiques américains. Les juristes nazis se croyaient en effet à l'aube d'une nouvelle ère fondée sur la race. L'idée était au fond de se rassurer en s'inspirant du modèle américain en particulier pour les plus radicaux des juristes. Il était important de se référer au modèle des États-Unis, le pays doté de la législation raciste par excellence.

Vous évoquez à la fin de votre ouvrage la situation du courant réaliste américain, en montrant que certains de ses présupposés (dépassement du formalisme, insistance sur la réalité de l'expérience juridique) sont largement partagés au-delà de ce courant dans la première moitié du $X^{\circledR}$ siècle et que l'attitude même des juristes nazis part d'un certain nombre de présupposés similaires. Que pouvez-vous nous dire sur le mouvement réaliste et ses liens avec les questions de race?

Le réalisme aux États-Unis est toujours très important. La plupart des juristes américains sont réalistes, y compris au sein du mouvement Law and Economics. Les autres traditions doctrinales ont perdu leur influence intellectuelle au sein des facultés de droit américaines. On croit que le droit, à travers la figure du juge, constitue le produit de forces sociales, voire, pour le dire dans les mots de Llewellyn, répond au "sens de la situation " ${ }^{14}$. Je suis moi-même proche du mouvement réaliste. Mais, en menant mes recherches sur ce livre, je me suis rendu compte à quel point l'attachement à la dogmatique est un élément important de la construction de l'État de droit.

$\mathrm{Au}$ fond, l'attachement aux traditions de forme ou, pour employer une image médiévale, aux brocards, c'est-à-dire aux formules qui intègrent les raisonnements

14. Karl N. Llewellyn, The Common Law Tradition: Deciding Appeals, Boston : Little Brown \& Co, 1960. 
juridiques tout en laissant ouverte l'interprétation, permet de maintenir le sens profond de la norme. Les vrais réalistes américains au contraire estiment, par exemple, qu'il faudrait d'abord se soucier du seul fonctionnement et de la dynamique du marché, pour ensuite adapter les normes à ce fonctionnement. Ces traditions sont très importantes aux États-Unis. Elles font la part belle au progressisme, à la gauche. Or, au sein du réalisme, les deux extrêmes se touchent. Roscoe Pound par exemple, s'il a été un temps progressiste, a aussi été un grand admirateur du système juridique nazi. C'est un point inquiétant de nos jours, cette manière de penser l'absence d'étanchéité du droit et de la politique et de la société, comme si ces dernières devaient entièrement conditionner la rationalité du premier.

C'est ce qui s'est donné à voir avec la nomination à la Cour suprême de Brett Kavanaugh, qui a suscité une tribune de plus de 3000 universitaires contre sa nomination. Ce dernier a tenu un discours politique dénonçant la conjuration des démocrates et des époux Clinton. Rarement, un tel degré de politisation a été assumé par un candidat à la Cour suprême. Mais si l'on y regarde bien cependant, le droit américain est sujet à ce genre de politisation depuis le XIX siècle. L'épisode Brett Kavanaugh ne constitue qu'une manifestation récente de ce processus de long terme. Cette politisation est liée à l'émergence du réalisme et à sa volonté de briser les frontières entre le droit et la société ou la politique. L'aboutissement d'une telle porosité s'est donné à voir du côté progressiste dans le mouvement des Critical Legal Studies dont sont issues les CRT. Je ne peux qu'éprouver une certaine sympathie pour les $C L S$ et ce qu'elles représentent. Mais, d'un autre côté, il faut reconnaître que l'épisode de la nomination de Brett Kavanaugh exprimant publiquement l'idée qu'il pourrait prendre, en tant que juge, des mesures de rétorsion contre les démocrates, puise en partie dans cette idée désormais assumée qu'il n'existe plus de frontières entre le droit et la politique. Le comportement public de Kavanaugh brise l'illusion d'une neutralité apparente de la justice et de ses représentants.

Du côté de l'Allemagne nazie, mon livre met en scène l'opposition entre nazis radicaux et nazis modérés. Il ne s'agit pas pour moi de défendre les modérés par rapport aux radicaux, mais d'observer que les modérés, du fait de leur formation, étaient davantage attachés au respect d'un certain nombre de formes juridiques traditionnelles. Si Lösner était un raciste antisémite, il demeurait attaché au principe de présomption d'innocence ou à une définition plus précise et claire de la race. Autant de principes qui auraient peut-être permis de mieux encadrer l'effet des lois de Nuremberg.

La fin de l'ouvrage compare le "Nazi realism " et l'" American realism ». Il a été montré, à l'inverse de ce qu'a soutenu Radbruch après la fin de la Seconde Guerre mondiale, que les juristes nazis rejetaient le positivisme et défendaient une conception concrète qui admettait largement la porosité des frontières entre droit et politique ou droit et société. Les juristes nazis, en recherchant le contact avec une certaine réalité au détriment des principes traditionnels du droit, sembleraient ainsi, à vous suivre, reprendre plusieurs des présupposés que l'on retrouve dans le réalisme juridique américain. $N$ 'y a-t-il pas alors une sorte de simple renversement de la "formule de Radbruch", consistant à dire que ce n'est pas le positivisme, mais 


\section{le réalisme des juristes nazis et leur recherche d'un droit concret qui expliquent le développement du droit nazi?}

Il ne s'agit évidemment pas de dire que le réalisme a été la cause du nazisme. C'est en 1946 que Radbruch, juriste resté en Allemagne pendant la période nazie, mais non rallié au régime, écrit un article qui s'attaque au positivisme ${ }^{15}$. Selon lui, les juristes nazis auraient été marqués par un positivisme idéologique qui leur aurait fait perdre toute référence au droit naturel et toute interrogation sur la justice de leurs actes. Mais cet article très court cite des exemples qui ont très peu à voir avec le positivisme. L'a-t-il fait dans le but de défendre la tradition juridique allemande ? Quoi qu'il en soit, cet article a été très utilisé dans les années 1950 en Allemagne, mais aussi aux États-Unis, pour suggérer que le problème à l'époque nazie a été la perte du contact avec le droit naturel. Je trouve que ces références à la « nature " sont toujours très ambiguës. Elles peuvent tout à fait faire référence à ce qui est considéré comme extérieur au droit, la politique ou la société.

Bernd Rüthers l'a montré dès les années 1960 : ce qui est en jeu dans le droit nazi est plutôt l'interprétation sans bornes du droit, en fonction des principes nazis 16 . Dans le régime nazi, l'homme de la "rue » doit toujours obéir sans discuter. Mais les Beamte, les agents publics, doivent se dire lorsqu'ils appliquent le droit: que ferait le Führer à ma place et comment interpréter en ce sens le droit ? Entre les mains des nazis, cette indétermination du droit - au sens d'antipositivisme - s'apparente avec la recherche du sens de la justice que le Führer aurait souhaité. Il y a, à bien y réfléchir, une certaine analogie avec le mode de raisonnement réaliste, qui insiste sur le rôle du juge et la possibilité de modifier le résultat par l'interprétation. Évidemment, l'arrière-plan des principes est très différent, mais il s'agit d'idées internationales qui prennent ensuite des formes nationales (allemandes, etc.).

Olivier Jouanjan termine son ouvrage récent sur le droit nazi 17 par la défense d'un certain formalisme du droit, entendu comme la garantie et la protection des individus et comparable au rule of law. Mais toute la question est de savoir ce que peut signifier le formalisme. Il est tout de même important de savoir, lorsqu'on se réclame du formalisme, en quoi ce formalisme peut être légitime. Un historien du droit se doit de ne pas faire table rase du contexte temporel qui préside à l'application des normes et principes juridiques.

Quelle a été la réception de votre dernier livre, Le modèle juridique d'Hitler, aux États-Unis ? Comment l'interprétez-vous?

Le livre visait à sensibiliser mes collègues américains. Je me suis attendu à des critiques qui ne sont finalement pas venues. Il est vrai que j'ai tenté d'apporter le maximum de soin à l'écriture de ce texte, afin d'anticiper le plus possible en amont

\footnotetext{
15. Gustav RADBRUCH, " Gesetzliches Unrecht und übergesetzliches Recht », Süddeutsche Juristenzeitung, 1, 1946, p. 105-108.

16. Bernd RÜTHERS, Die Unbegrenzte Auslegung. Zum Wandel der Privatrechtsordnung im Nationalsozialismus, Tübingen : Mohr Verlag, 1968.

17. Olivier JoUANJAN, Justifier l'injustifiable. L'ordre du discours juridique nazi, Paris : PUF, coll. « Leviathan », 2017.
} 
les critiques qui auraient pu m'être adressées. Je savais qu'il s'agissait d'un sujet polémique à plus d'un titre.

Le plus inquiétant est qu'à la sortie du livre, j'ai été contacté par un groupe de nazis américains, qui ont beaucoup apprécié le livre. Ils ont voulu que je contribue à leur site, ce à quoi je me suis naturellement opposé. J'ai aussi été approché pareillement par un groupe de communistes. Cela mis à part, je peux dire que le livre a été globalement bien accueilli aux États-Unis, sans doute aussi parce que j'ai voulu limiter la part de la réflexion théorique et philosophique. Je me suis au contraire attaché à rendre compte des faits, même s'il y a toujours bien sûr un effort minimal de réflexion et de conceptualisation. Le débat suscité après la publication du livre aux États-Unis était d'ordre moral. La question était de savoir à quel point la nation américaine s'est-elle souillée vis-à-vis du nazisme?

La réception du livre a été moins bonne en Allemagne. Certains comptes rendus ont été favorables, en particulier celui de Michael Stolleis ${ }^{18}$. Mais, dans le champ médiatique, j'ai été attaqué par un éditorialiste du journal Die Zeit soutenant que les thèses développées dans mon livre contribuaient à minorer la responsabilité des nazis dans l'idéologie raciste. Mon propos n'était toutefois pas destiné en priorité au public allemand, mais américain. Il consistait à dire que les lois de Nuremberg s'intégraient au sein d'une matrice idéologique plus large que celle du régime nazi. Je ne nie pas le risque que l'extrême droite allemande soit tentée d'instrumentaliser les thèses de mon livre pour minimiser l'influence du régime nazi dans la production des lois de Nuremberg. Mais, en dehors des spécialistes, le livre a été accueilli de manière relativement silencieuse en Allemagne. Sans doute met-il encore mal à l'aise une partie de l'opinion publique allemande, à l'inverse de celle des ÉtatsUnis, qui y a trouvé un intérêt important.

\section{- L'auteur}

James Q. Whitman est Ford Foundation Professor of Comparative and Foreign Law à l'Université de Yale, aux États-Unis. Il a fait ses études à l'Université de Columbia, à l'Université de Chicago et à l'Université de Yale. Il a également enseigné à l'Université de Stanford et été invité dans plusieurs universités américaines et européennes. Ses recherches portent en particulier sur l'histoire du droit, non seulement américaine, mais aussi européenne.

Entretien réalisé par Guillaume Richard, professeur d'histoire du droit à l'Université Paris V Paris-Descartes, IHD (EA 2515), et Lionel Zevounou, maître de conférences en droit public à l'Université Paris-Nanterre, CTAD (UMR 7074), membre junior de l'Institut Universitaire de France.

18. Michael STOLLEIS, « Lehrmeister America », Rechtsgeschichte, 26, 2018, p. 489-492. 